\title{
Comparação da insatisfação corporal e do comportamento alimentar inadequado em atletas adolescentes de diferentes modalidades esportivas
}

CDD. 20.ed. 302.222

613.7

\author{
Leonardo de Sousa FORTES* \\ Maria Elisa Caputo FERREIRA*
}

*Universidade Federal de Juiz de Fora.

\begin{abstract}
Resumo
A insatisfação corporal (IC) é considerada sintoma de primeira ordem no desencadeamento dos transtornos alimentares (TAs). 0 ambiente atlético tem fatores peculiares que parecem potencializar o risco do surgimento de TAs nos atletas. 0 objetivo do presente estudo foi comparar a IC e o comportamento alimentar inadequado (CAI) entre atletas adolescentes de diversas modalidades esportivas e diferentes niveis competitivos, utilizando o índice de massa corporal (IMC), o percentual de gordura e a idade como covariáveis. A amostra do estudo foi constituida por 580 atletas de diversas modalidades, de ambos os sexos, com idades entre 10 e 19 anos. Utilizou-se o BSO e o EAT-26 para mensurar a IC e o CAI, respectivamente. Peso e estatura foram aferidos para calcular-se o IMC. A gordura relativa foi estimada aferindo-se as dobras cutâneas triciptal e subescapular. Foi conduzida análise univariada de covariância (ANCOVA) para comparar IC e CAI entre niveis competitivos e modalidades esportivas, segundo sexo. Em relação aos resultados, dois aspectos merecem destaque. 0 primeiro é que identificou-se diferença de IC entre basquetebolistas e handebolistas do sexo masculino $(p<0,05)$. Já o segundo ponto a ser destacado, é a diferença encontrada de CAl entre voleibolistas e atletas de nado sincronizado do sexo feminino $(p<0,05)$. Os resultados do presente estudo permitem concluir que o padrão alimentar de esportistas adolescentes, praticantes de modalidades com características distintas, foi semelhante, havendo diferenças em poucas modalidades esportivas.
\end{abstract}

UnITERMOS: Imagem corporal; Transtornos alimentares; Esporte.

\section{Introdução}

Apesar, de nos últimos anos, ter crescido o interesse pelo estudo de transtornos alimentares (TAs) em atletas, existe ainda um entendimento pouco claro sobre o impacto desse problema em contextos esportivos (Sundgot-Borgen \& Torstveit, 2004). De fato, quando analisamos a investigação sobre modalidades esportivas consideradas de risco para psicopatologias em atletas, os dados são ainda muito discrepantes, existindo estudos que apontam valores residuais (BAUM, 2006; Denoma, Scaringi, Gordon, Van Orden \& Joiner, 2009; Perini, Vieira, Vigário, Oliveira, Ornellas \& Oliveira, 2009; Schaal, Tafflet, Nassif, Thibault, PIChard, Alcote, Guillet, Berthelot, Simon \& TousSAINT, 2011;TORSTVEIT \& SUNDGOT-BORgEN, 2005).

Devido a esta disparidade de resultados, alguns autores (Schaal et al., 2011; Vieira, Oliveira, Vieira,
Vissoci, Hoshino \& Fernandes, 2009) tem vindo a chamar a atenção para a necessidade dos estudos assumirem um "carácter mais utilitário", comparando fatores psicológicos que possam predispor a TAs entre modalidades esportivas com características distintas.

Um desses fatores, é a imagem corporal. Segundo SLADE (1988), ela diz respeito a aparência mental que temos de nosso corpo. A imagem corporal é um fenômeno biopsicocultural que possui vários aspectos associados em sua formação, tais como: crenças, pensamentos, desejos e investimentos que se tem com o próprio corpo (Conti, Frutuoso \& Gambardella, 2005). A insatisfação corporal faz parte de um subcomponente da dimensão atitudinal da imagem corporal e diz respeito à depreciação que o sujeito tem com sua aparência física (Lepage \& 
CROWTHER, 2010). Autores alegam que a insatisfação corporal é considerada sintoma de primeira ordem no desencadeamento dos TAs (BONCI, BonCI, GRANGER, Johnson, Malina, Milne, Ryan \& Vanderbunt, 2008; Conti, Frutuoso \& Gambardella, 2005).

TAs como a Anorexia Nervosa (AN), caracterizada por restrição alimentar, medo mórbido de engordar, baixo peso corporal, distorção da imagem corporal e uso de medicamentos para emagrecimento (Perini et al., 2009), e a Bulimia Nervosa (BN) que apresenta como sintomas o uso de comportamentos compensatórios como a prática excessiva de atividade física e/ou indução de vômitos após um episódio de alimentação exagerada (PERINI et al., 2009), são os mais comumente encontrados na população (BAUM, 2006), principalmente entre adolescentes do sexo feminino (Torstveit \& Sundgot-Borgen, 2005). Evidências científicas mostram que o ambiente atlético tem fatores peculiares que parecem potencializar o risco do surgimento de TAs nos atletas (BAUM, 2006; Perini et al., 2009). Pressão de treinadores e pais no anseio por melhores resultados, vestimentas que salientam a forma corporal, ênfase dada a magreza e perda de peso corporal são alguns deles (BAUM, 2006). Além disso, modalidades que exigem do atleta leveza de movimentos, baixa percentagem de gordura corporal e baixo peso corporal, ou aquelas que são divididas por categorias de peso, ou ainda as que dependem de avaliações externas feitas por juízes

\section{Metodologia}

\section{Aspectos éticos}

O estudo obteve aprovação do comitê de ética e pesquisa envolvendo seres humanos da Universidade Federal de Juiz de Fora com o parecer número 232/2010, de acordo com a resolução 196/1996. O Termo de Consentimento Livre e Esclarecido foi assinado pelos pais dos atletas explicando todos os procedimentos da pesquisa, além de garantir anonimato aos participantes e total sigilo no tratamento dos dados.

\section{População e amostra}

A população do estudo foi constituída por atletas adolescentes competitivos com idades de 10 a 19 anos, residentes em cidades dos Estados do Rio de Janeiro e Minas Gerais no Brasil. Para seleção da amostra, foi adotado o critério de amostragem são consideradas de risco para o desencadeamento de TAs (Denoma et al., 2009). São elas: ginástica artística, ginástica rítmica, "triathlon", nado sincronizado, saltos ornamentais, judô, "taek-won-do", esgrima, karatê, remo, patinação artística, entre outras.

Além do tipo de modalidade esportiva, o nível competitivo também possui uma forte associação com o aparecimento de alimentação desordenada (Gomes \& Silva, 2010). Baum (2006) e Denoma et al. (2009) argumentam que atletas de alto nível competitivo podem apresentar maiores riscos para TAs. Apesar destas suposições e hipóteses, os estudos já realizados não têm sido totalmente conclusivos quanto aos seus resultados, deixando ainda uma lacuna do conhecimento, se de fato, atletas de modalidades consideradas de "alto risco" são mais insatisfeitos e se estes apresentam maiores índices de comportamentos alimentares inadequados que atletas de esportes ditos de "baixo risco" para tais desfechos. Além disso, não foi encontrado na literatura estudo que tenha comparado estas variáveis entre mais de quatro modalidades esportivas e entre diferentes níveis competitivos.

Diante das reflexões acima, o objetivo do presente estudo foi comparar a insatisfação corporal e o comportamento alimentar inadequado entre atletas adolescentes de diversas modalidades esportivas e diferentes níveis competitivos, utilizando o índice de massa corporal, o percentual de gordura e a idade como covariáveis. casual simples descrita por Perini et al. (2009). Os atletas provinham de clubes das cidades de Juiz de Fora/MG, Barbacena/MG, Três Rios/ $\mathrm{RJ}$ e Rio de Janeiro/RJ, clubes estes pertencentes aos campeonatos nacionais, estaduais e regionais das modalidades avaliadas. Inicialmente, foram incluídos no estudo 620 atletas de ambos os sexos, porém, 40 destes sujeitos foram excluídos da amostra por não responderem os questionários em sua totalidade, chegando a uma amostra final de 580 atletas de diversas modalidades esportivas (atletismo, basquete, esgrima, futebol, ginástica artística, handebol, judô, nado sincronizado, natação, polo aquático, saltos ornamentais, "taek-won-do", "triathlon" e voleibol). O tamanho amostral por nível competitivo, modalidade e sexo está elucidado na TABELA 1. Para inclusão de sujeitos na pesquisa, foram adotados os seguintes critérios: 
1 ) Apresentar o Termo de Consentimento Livre e Esclarecido assinado pelo responsável.

2) Estar em processo de treinamento físico sistematizado com frequência mínima de três dias semanais e duração mínima de $1 \mathrm{~h} /$ treino.

3) Ter participação mínima de uma competição de dimensão regional no ano de 2011.

4) Ter disponibilidade para responder questionários.

\section{Instrumentos e medidas}

Para avaliar o comportamento alimentar inadequado foi aplicado o "Eating Attitudes Test" (EAT-26). Este é um questionário com 26 questões que avaliam recusa patológica alimentar, preocupação exacerbada com aparência física, comportamentos purgativos, influência do ambiente na ingestão alimentar e autocontrole sobre os alimentos. A versão utilizada para o sexo feminino foi validada por Bighetti, Santos, SANTOS e Ribeiro (2004), apresentando consistência interna de 0,82. Em adolescentes do sexo masculino, a versão aplicada foi a de Fortes, AmAral, Conti e Ferreira (submetido). No estudo de Fortes et al. (submetido), o Alfa de Cronbach encontrado foi de 0,87 e não foi identificada diferença entre os escores do EAT-26 no teste-reteste, apresentando então uma boa reprodutibilidade do questionário para esta população. A pontuação é feita pela soma dos itens. Escore igual ou maior que 20 representa indivíduos que apresentam comportamento alimentar de risco para TAs. Existem seis opçóes de resposta que variam de 0 a 3 pontos (sempre $=3$, muitas vezes $=2$, frequentemente $=1$, poucas vezes $=0$, quase nunca $=0 \mathrm{e}$ nunca $=0$ ). A única questão que apresenta pontuação em ordem invertida é a 25.

Para avaliar a insatisfação corporal foi aplicado o "Body Shape Questionnaire" (BSQ). Trata-se de um teste de autopreenchimento com 34 perguntas que procura avaliar a preocupação que o sujeito apresenta com seu peso e com sua aparência física. A versão utilizada foi validada para adolescentes brasileiros (CONTI, CORDÁs \& LATORRE, 2009) e sua análise de consistência interna revelou um $\alpha$ de 0,96 para ambos os sexos e um coeficiente de correlação entre os escores do teste-reteste significativo, variando de 0,89 a 0,91 para meninas e meninos, respectivamente. $\mathrm{O}$ escore é dado pela soma dos itens, que classifica níveis de insatisfação a respeito do corpo, sendo: $<80$ pontos livre de insatisfação corporal, entre 80 e 110 leve insatisfação, entre 110 e 140 insatisfação moderada e pontuações acima de 140 grave insatisfação corporal, ou seja, quanto maior o escore, maior a insatisfação com o corpo.
Foi aplicado um questionário qualitativo para avaliar dados demográficos como: idade, sexo, modalidade esportiva e nível competitivo.

Foram aferidos dobras cutâneas, peso e estatura. Os procedimentos para as coletas destas variáveis foram realizados de acordo com as padronizaçōes determinadas pela ISAK (2001).

O percentual de gordura ( $\% \mathrm{G})$ foi estimado pelo método duplamente indireto. Foram mensuradas as dobras cutâneas triciptal e subescapular. Estas medidas foram tomadas de forma rotacional e coletadas três vezes, sendo considerada a média dos valores. Para cálculo do \%G foi utilizado o protocolo de Slaughter, Lohman, Boileau, Hoswill, Stillman e YALOAN (1988) criado para adolescentes.

A massa corporal foi mensurada por uma balança digital portátil da marca Tanita com precisão de 100 g e capacidade máxima de $200 \mathrm{~kg}$. Foi utilizado estadiômetro portátil com precisão de $0,1 \mathrm{~cm}$ e altura máxima de 2,20 m da marca Welmy para mensurar a estatura dos atletas.

$\mathrm{O}$ índice de massa corporal [IMC = massa corporal $(\mathrm{kg}) /$ estatura $\left.\left(\mathrm{m}^{2}\right)\right]$ foi usado como indicador de estado nutricional. A classificação do IMC obedeceu aos critérios da WHO (2007), que propõe a classificação em baixo peso, peso normal, sobrepeso e obeso, de acordo com percentis $(5,85$ e 95$)$ por idade cronológica.

\section{Procedimentos}

Os atletas foram abordados em seus locais de treinamento mediante autorização dos treinadores responsáveis pelas equipes. Foi explicado de forma sucinta o tema do estudo e o propósito do mesmo, bem como todo procedimento necessário para integrá-los na amostra da pesquisa. Após este primeiro momento, os sujeitos levaram para seus responsáveis o Termo de Consentimento Livre Esclarecido, sendo orientados a devolverem devidamente assinado, entregando-o nas mãos de seu treinador na semana subsequente.

Foram realizados dois encontros em dias consecutivos com todos os atletas. O primeiro era destinado à aplicação dos questionários EAT-26 e BSQ, e o segundo, foi utilizado para mensurar medidas antropométricas em todos os participantes do estudo. Todos os clubes que fizeram parte do presente estudo, disponibilizaram uma sala adequada para aplicação dos questionários e realizar avaliação antropométrica.

Os questionários foram entregues aos atletas, que receberam então a mesma orientação verbal. Uma orientação escrita sobre os procedimentos adequados também estava presente nos mesmos. As eventuais 
dúvidas foram esclarecidas pelos responsáveis pela aplicação do BSQ e EAT-26. Os sujeitos do estudo não se comunicavam entre si. A distribuição dos instrumentos foi efetuada no momento que os atletas adentravam no ambiente (sala), sendo seu preenchimento de caráter voluntário. Não houve limite de tempo para preenchê-los.

As medidas das dobras cutâneas foram realizadas pelo mesmo avaliador para aumentar a fidedignidade da avaliação. Mesmo assim, as medidas podem ser afetadas pela variabilidade intra-avaliador. A literatura recomenda que não ocorram diferenças superiores a $10 \%$ entre uma medida e as demais no mesmo local, por isso foi realizado o cálculo do erro técnico de medida (ETM), proposto por Perini, Oliveira, Ornellas e Oliveira (2005), excluindo dados com variância maior que $10 \%$.

\section{Análise dos dados}

Utilizou-se a estatística descritiva (média, desvio padrão) para as variáveis comportamento alimentar

\section{Resultados}

A maioria dos atletas do estudo eram do sexo masculino (80\%), participantes de modalidades consideradas de baixo risco para TAs $(84,65 \%)$ e competiam em nível regional $(45,86 \%)$ (TABELA 1$)$. No total, foram avaliadas 14 modalidades esportivas, sendo que 12 incluíam atletas do sexo masculino e oito apresentavam meninas atletas, como pode ser observado na TABELA 1.

A TABELA 2 apresenta a comparação das pontuações do BSQ e do EAT-26 entre grupos de sexo, modalidades e nível competitivo. Foi identificada diferença de insatisfação corporal entre atletas masculinos e femininos $(p<0,05)$. Além disso, atletas que competiam em âmbito regional apresentaram maiores pontuações no EAT-26 comparado aos demais grupos de nível competitivo $(\mathrm{p}<0,05)$. Não encontrou-se diferença para estas inadequado (EAT-26) e insatisfação corporal (BSQ). Foram agrupadas modalidades esportivas consideradas de risco para TAs segundo KRENTZ e WASCHBURGER (2011) (esgrima, ginástica artística, judô, nado sincronizado, saltos ornamentais, "taekwondo" e "triathlon") no grupo "modalidades de risco", e o restante, foram integradas no grupo "modalidades sem risco". Foi conduzida análise univariada de covariância (ANCOVA), para comparar insatisfação corporal e comportamento alimentar inadequado entre grupos de sexo (feminino e masculino), grupos de modalidades (modalidades de risco e modalidades sem risco), grupos de níveis competitivos (regional, estadual e nacional) e entre todas as modalidades esportivas, sempre utilizando o \% $\mathrm{G}$, IMC e idade como covariáveis. O “post hoc" de Bonferroni foi utilizado para identificar tais diferenças. Todos os dados foram analisados no "software" SPSS 17.0, considerando-se o nível de significância de 5\%.

variáveis na comparação entre os demais grupos por intermédio da análise de ANCOVA.

Em relação às comparações de insatisfação corporal e comportamento alimentar inadequado entre as modalidades esportivas de acordo com o sexo, utilizando o IMC, percentual de gordura e idade como covariáveis na análise univariada de covariância, dois aspectos merecem destaque. O primeiro é que identificou-se diferença de insatisfação corporal entre basquetebolistas e handebolistas do sexo masculino $(\mathrm{p}<0,05)$. Já o segundo ponto a ser destacado, é a diferença encontrada de comportamento alimentar inadequado entre voleibolistas e atletas de nado sincronizado do sexo feminino ( $\mathrm{p}$ $<0,05)$. Estas comparações podem ser visualizadas na TABELA 3. 
TABELA 1 - Distribuição de frequência de atletas adolescentes competitivos brasileiros por modalidade, sexo, grupo de modalidade e nível competitivo.

\begin{tabular}{|c|c|c|c|c|c|c|c|c|c|c|c|c|c|c|c|}
\hline 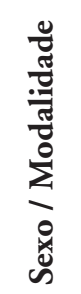 & 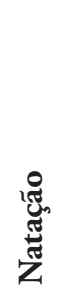 & 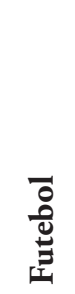 & $\begin{array}{l}\overline{0} \\
\overline{8} \\
\overline{0} \\
\bar{\Xi} \\
\bar{\Xi}\end{array}$ & $\begin{array}{l}\overline{0} \\
\overline{0} \\
\overline{0} \\
\bar{z} \\
\bar{z} \\
0\end{array}$ & $\begin{array}{l}\overline{0} \\
\frac{0}{0} \\
\frac{0}{\circ}\end{array}$ & 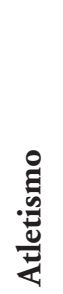 & 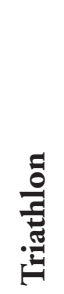 & $\stackrel{\varrho}{\Xi}$ & 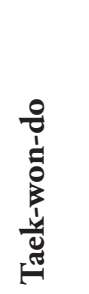 & 蛋 & 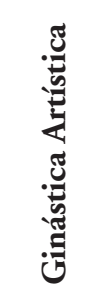 & 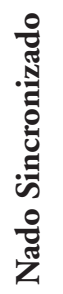 & 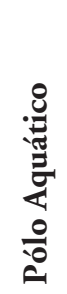 & 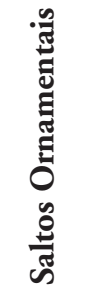 & हैँّ \\
\hline M & 42 & 271 & 16 & 56 & 6 & 10 & 14 & 14 & 6 & 8 & - & - & 16 & 5 & 464 \\
\hline $\mathrm{F}$ & 22 & - & 20 & 16 & 16 & - & - & - & 5 & - & 14 & 18 & - & 5 & 116 \\
\hline \multicolumn{4}{|c|}{ Grupo } & \multicolumn{4}{|c|}{ MR } & \multicolumn{5}{|c|}{ MSR } & \multicolumn{3}{|c|}{ Total } \\
\hline \multicolumn{4}{|c|}{ Frequência } & \multicolumn{4}{|c|}{89} & \multicolumn{5}{|c|}{491} & \multicolumn{3}{|c|}{580} \\
\hline \multicolumn{4}{|l|}{ NC } & \multicolumn{3}{|c|}{ Regional } & \multicolumn{3}{|c|}{ Estadual } & \multicolumn{3}{|c|}{ Nacional } & \multicolumn{3}{|c|}{ Total } \\
\hline \multicolumn{4}{|c|}{ Frequência } & \multicolumn{3}{|c|}{266} & \multicolumn{2}{|r|}{129} & & \multicolumn{3}{|c|}{185} & \multicolumn{3}{|c|}{580} \\
\hline
\end{tabular}

NC = Nível Competitivo; $\mathrm{MR}=$ "Modalidades de risco";

MSR $=$ "Modalidades sem risco"; $M=$ Masculino $\mathrm{F}=$ Feminino

TABELA 2 - Comparação da insatisfação corporal e do comportamento alimentar inadequado entre grupos de níveis competitivos, grupos de sexo e grupos de modalidades.

\begin{tabular}{lccc}
\hline Variável / Grupo & Regional & Estadual & Nacional \\
\hline BSQ & $63,58 \pm 1,37$ & $60,89 \pm 1,93$ & $63,28 \pm 1,64$ \\
EAT-26 & $13,40 \pm 0,67 \dagger \neq$ & $9,70 \pm 0,95^{*}$ & $10,49 \pm 0,80^{*}$ \\
& & & \\
Variável / Grupo & Feminino & Masculino & \\
BSQ & $74,41 \pm 2,11 \dagger$ & $60,01 \pm 1,00^{*}$ & \\
EAT-26 & $11,26 \pm 1,08$ & $11,75 \pm 0,51$ & \\
& & & \\
Variável / Grupo & Modalidades de Risco & Modalidades Sem Risco & \\
BSQ & $66,54 \pm 2,36$ & $62,23 \pm 0,98$ & \\
EAT-26 & $10,41 \pm 1,17$ & $11,87 \pm 0,49$ & \\
\hline
\end{tabular}

p $<0,05$ em relação ao Regional;

${ }^{\dagger} p<0,05$ em relação ao Estadual;

${ }^{\ddagger} p<0,05$ em relação ao Nacional;

* $p<0,05$ em relação ao Feminino;

${ }^{\dagger} p<0,05$ em relação ao Masculino. 
TABELA 3 - Comparação da insatisfação corporal e do comportamento alimentar inadequado entre modalidades esportivas de acordo com o sexo em atletas adolescentes competitivos.

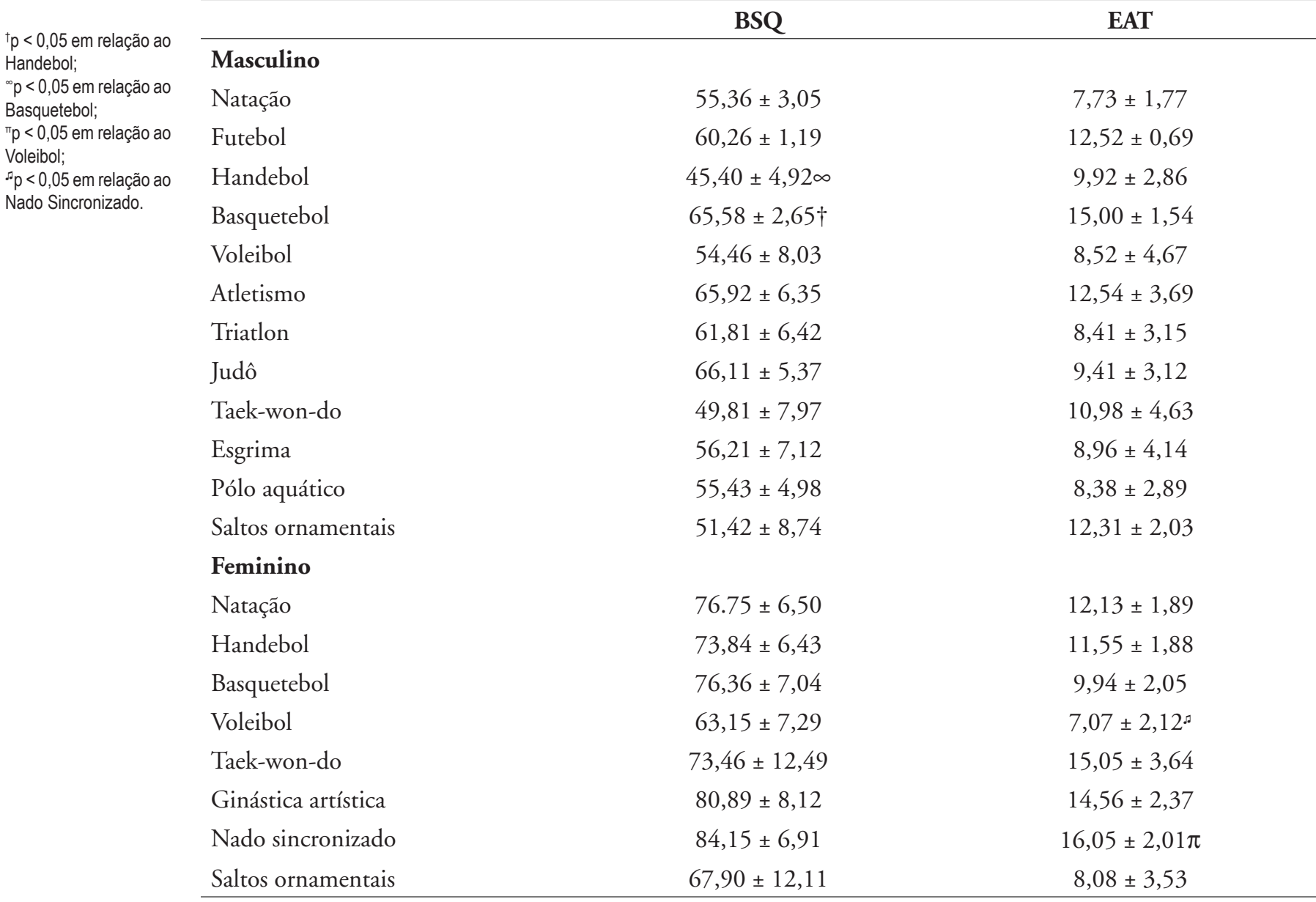

\section{Discussão}

Este estudo comparou a insatisfação corporal e o comportamento alimentar inadequado entre atletas adolescentes brasileiros, com diferentes níveis competitivos, de ambos os sexos e de modalidades esportivas com características distintas. Para estas análises, procurou-se igualar as covariáveis (IMC, $\% \mathrm{G}$ e idade) entre as modalidades esportivas, para que não houvesse influência destas sobre as variáveis dependentes, neste caso, nos referimos à insatisfação corporal e o comportamento alimentar inadequado. Estes resultados mostraram que atletas adolescentes brasileiros competitivos do sexo feminino eram mais insatisfeitos com o corpo do que seus pares $(\mathrm{p}<$ $0,05)$ (TABELA 2). Atletas que competiam em nível regional apresentaram maior comportamento alimentar inadequado comparado aos demais grupos de nível competitivo $(\mathrm{p}<0,05)$ (TABELA 2). Basquetebolistas apresentaram-se mais insatisfeitos com o corpo do que handebolistas do sexo masculino $(\mathrm{p}<0,05)$, e atletas de nado sincronizado do sexo feminino mostraram ter um comportamento alimentar mais desordenado que as voleibolistas $(\mathrm{p}<0,05)$ (TABELA 3).

Estudos têm apresentado que meninas adolescentes são mais acometidas por insatisfação com peso e aparência corporal do que os meninos da mesma faixa etária (Conti, Frutuoso \& Gambardella, 2005; Martins, Pelegrini, Matheus \& Petroski, 2010), independente de serem atletas ou não (Hausenblas \& Downs, 2001; SChaAl et al., 2011). Os achados do presente estudo replicaram esta tendência, já que foi identificado que atletas do sexo feminino apresentaram maiores pontuaçôes no BSQ, havendo diferença significativa $(\mathrm{p}<0,05)$ de insatisfação corporal comparado aos meninos.

Autores afirmam que o nível competitivo acentuado pode provocar maior preocupação com peso e 
aparência física em atletas (BAUM, 2006; PERINI et al., 2009). Segundo Denoma et al. (2009) e SungotBORGEN e TORSTVEIT (2004), atletas com maior nível competitivo sofrem maiores pressōes de treinadores, patrocinadores, familiares, amigos, portanto, estes indivíduos, parecem apresentar maior preocupação com características morfológicas, no intuito de se obter um melhor rendimento esportivo. No entanto, De Bruin, OUdejANS e BAKKER (2007), identificaram que ginastas que competiam em níveis menores eram mais preocupadas com peso corporal do que atletas de ginástica da elite alemã. Por outro lado, Gomes e SiLva (2010) não encontraram diferenças de preocupação com aparência nos diferentes níveis competitivos em atletas portugueses de ambos os sexos. Os resultados do presente estudo corroboram com os achados de Gomes e Silva (2010), pois também não foi encontrada diferença de insatisfação corporal entre os diferentes grupos de nível competitivo.

Esportes que preconizam baixo percentual de gordura e baixo peso corporal, também parecem acentuar a insatisfação corporal entre atletas (KRENTZ \& WARCHSBURGuer, 2011; Perint et al., 2009). Em um estudo que avaliou a influência de diversas variáveis sobre a insatisfação com o corpo em atletas de elite, SCOFFIER, Maiano e D'ArRIPE-LongueVILle (2010) por meio de uma análise multivariada de variância (MANOVA), identificaram que a participação em modalidades esportivas de estética respondeu a $68 \%$ da variância da dimensão de preocupação com aparência física. SCHAAL et al. (2011) avaliaram 2067 atletas franceses de 36 modalidades esportivas distintas. Estes autores identificaram que esportistas de modalidades com características estéticas e de modalidades esportivas com habilidades motoras finas apresentaram maior depressão, além de serem mais insatisfeitos com peso e aparência corpórea que atletas dos demais esportes. Alguns resultados da presente pesquisa são controversos em relação as questôes retromencionadas, já que não se encontrou diferença estatística de insatisfação corporal entre os grupos de modalidades de risco e sem risco (TABELA 2), além de atletas de basquetebol (modalidade coletiva) do sexo masculino apresentarem maior pontuação no BSQ sobre as demais modalidades, havendo, apenas, diferença para os handebolistas ( $\mathrm{p}<$ $0,05)$ (TABELA 3). Já em relação ao sexo feminino, o esperado era que atletas de modalidades de características estéticas (nado sincronizado, ginástica artística e saltos ornamentais) apresentassem maior insatisfação corporal que as demais modalidades esportivas. No entanto, não foram encontradas diferenças desta variável entre modalidades esportivas neste sexo (TABELA 3).
Alguns pesquisadores argumentam que a insatisfação corporal pode ser uma das variáveis preditoras para problemas psicológicos, como são os casos dos TAs (Bonci et al., 2008; Conti, Frutuoso \& GAMBARDELLA, 2005). Quando falamos de atletas, esta tendência parece ser diferente. HaUsENBLAS e Downs (2001), realizaram uma revisão da literatura comparando a imagem corporal de atletas e não atletas e mostraram que esportistas competitivos possuem esta mais positiva que os não atletas, porém, vários autores encontraram em seus estudos que atletas apresentam maiores riscos para TAs (PERINI et al., 2009; SCHAAL et al., 2011; Sundgot-Borgen \& TorstVeit, 2004).

Quando nos remetemos aos TAs, estudos tem apresentado que meninas possuem comportamento alimentar mais desordenado que os meninos (MARtins et al., 2010; Scherer, Martins, Pelegrini, Matheus \& Petroski, 2010). Esta tendência parece se repetir em atletas (VIEIRA et al., 2006; SUNDGOTBorgen \& TorstVert, 2004). Os achados do presente estudo não confirmaram esta tendência, pois não foi encontrada diferença de pontuações do EAT-26 entre atletas do sexo feminino e masculino (TABELA 2).

O nível competitivo parece ser um grande influenciador na predisposição para aumento de riscos de comportamento alimentar desordenado (Krentz \& Warchsburger, 2011; Perini et al., 2009; Sundgot-Borgen \& Torstveit, 2004), independente do sexo avaliado (Johnson, Crosby, Engel, Mitchell, Powers, Wittrock \& WonDerlich, 2004; Reiking \& Alexander, 2005). No entanto, ainda não existe um indicador claro para classificar nível competitivo em atletas (GoMES \& SiLvA, 2010). Geralmente são utilizados os seguintes métodos: participação em competições, horas de treino semanal, quantidade de medalhas adquiridas em campeonatos, entre outros. DenOMA et al. (2009), encontraram em seus achados, que quando o nível competitivo aumentava, era notado aumento da frequência de comportamentos bulímicos. Em uma pesquisa envolvendo grupos de ginastas com diferentes níveis competitivos, DE Bruin, Oudejans e BaKker (2007) identificaram que atletas de elite apresentaram maiores frequências de comportamentos de dieta e uso de métodos patogênicos para controle de peso, comparados aos grupos com menor nível de competitividade. Os resultados do presente estudo vão ao encontro dos achados descritos acima. Atletas com menor nível competitivo apresentaram maiores pontuações no EAT-26, havendo diferenças significativas $(\mathrm{p}<0,05)$ sobre os demais grupos (TABELA 2). 
Além do nível competitivo, KRENTZe WARSCHBURGER (2011) argumentam que são encontrados maiores riscos para TAs em atletas que praticam modalidades esportivas de cunho estético e/ou que preconizam baixo \%G e baixo peso corporal. O presente estudo não encontrou diferença de comportamento alimentar desordenado entre os grupos modalidades de risco e sem risco. De qualquer forma, ainda são raros os estudos que comparam comportamentos alimentares inadequados em diversas modalidades esportivas. Em um destes raros estudos, Filaire, Rouveix, Pannafieux e Ferrand (2007), mostraram que judocas utilizavam com maior frequência em relação a ciclistas, métodos patogênicos para controle de peso, tais como: indução de vômito, uso de diuréticos e pílulas para emagrecimento. SchaAl et al. (2011) identificaram que esportistas de modalidades estéticas e de habilidades motoras finas apresentaram com maior frequência, comportamentos alimentares desordenados em relação a atletas de esportes de equipe com bola, modalidades com uso de raquete e modalidades de combate. Os achados do presente estudo corroboram com esta tendência apenas no sexo feminino. Foi encontrada maiores pontuações no EAT-26 em meninas praticantes de nado sincronizado em relação as demais modalidades esportivas, porém, identificou-se diferença apenas para as voleibolistas $(\mathrm{p}<0,05)$ (TABELA 3).

\section{Conclusão}

No que diz respeito à insatisfação corporal, concluiu-se que basquetebolistas do sexo masculino eram mais insatisfeitos com o corpo que atletas de handebol. Por outro lado, não houve diferença desta variável afetiva nos diferentes grupos de nível competitivo, nem entre modalidades esportivas do sexo feminino.
Apesar deste ser o primeiro estudo brasileiro a ser desenvolvido comparando insatisfação corporal e comportamentos alimentares desordenados entre diversas modalidades esportivas, a presente pesquisa possui limitações. A principal limitação foi o baixo tamanho amostral para algumas modalidades esportivas (esgrima, saltos ornamentais e "taekwondo"). Este fato pode ter influenciado os resultados de alguma forma. No entanto, salientase a dificuldade de acesso a avaliaçốes deste gênero em atletas adolescentes brasileiros. No Brasil, ainda paira uma subcultura de desconfiança para tais avaliaçōes. Treinadores não reconhecem a importância deste tipo de estudo e geralmente negam a participação voluntária de sua equipe. Por isso, o avanço do conhecimento científico nesta área com esta população fica restrito. Portanto, são incentivados estudos com estas características para que se possa entender melhor variáveis comportamentais e afetivas em esportistas competitivos.

Sugere-se que estudos futuros comparem estas variáveis entre modalidades com características diferentes destas aqui avaliadas. Além disso, são sugeridos estudos que pesquisem a influência de indicadores antropométricos sobre a insatisfação corporal e comportamento alimentar desordenado em atletas.
Em relação ao comportamento alimentar de risco para TAs, constatou-se que as pontuações do EAT26 foram significativamente maiores em atletas que competiam em âmbito regional. Ademais, foram identificadas diferenças estatísticas desta variável comportamental entre as modalidades esportivas somente no sexo feminino.

\begin{abstract}
Comparison of body dissatisfaction and inappropriate eating behavior in adolescent athletes of different sports

The body dissatisfaction (BD) is considered a symptom of first order in the onset of eating disorders (ED). The athletic environment has unique factors that appear to potentiate the risk for the emergence of ED in athletes. The purpose of this study was to compare the BD and inappropriate eating behavior (IEB) among adolescent athletes of different sports and competitive levels using the body mass index (BMI), fat percentage and age as covariates. The study sample consisted of 580 athletes of both sexes, aged between 10 and 19 years. We used the BSO and the EAT-26 to measure the BD and IEB, respectively.
\end{abstract}


Weight and height were measured for BMI determination. Relative fat was estimated via triceps and subscapular skinfolds. A univariate analysis of covariance (ANCOVA) was conducted to compare BD and IEB between the different competitive levels and sports, according to sex. A significant differences in BD was observed between male basketball and handball players $(p<0.05)$. Additionally, a significant difference was found in IEB between female volleyball players and synchronized swimmers $(p<0.05)$. The results of this study allow us to conclude that the dietary patterns are similar in adolescent athletes regardless of the sport and competitive level.

UnITERMS: Body image; Eating disorders; Sport.

\section{Referências}

BAUM, A. Eating disorders in the male athlete. Sports Medicine, Auckland, v.36, n.1, p.1-6, 2006.

BIGHETTI, F.; SANTOS, C.B.; SANTOS, J.E.; RIBEIRO, R.P.P. Tradução e avaliação do Eating Attitudes Test em adolescentes do sexo feminino de Ribeirão Preto, São Paulo. Jornal Brasileiro de Psiquatria, Rio de Janeiro, v.53, n.6, p.339-46, 2004.

BONCI, C.M.; BONCI, L.J.; GRANGER, L.R.; JOHNSON, C.L; MALINA, R.M.; MILNE, L.W.; RYAN, R.R.; VANDERBUNT, E.M. National athletic trainers' association position statement: preventing, detecting, and managing disordered eating in athletes. Journal of Athletic Training, Dallas, v.43, n.1, p.80-108, 2008.

CONTI, M.A.; CORDÁS, T.A.; LATORRE, M.R.D.O. Estudo de validade e confiabilidade da versão brasileira do body shape questionnaire (BSQ) para adolescentes. Revista Brasileira de Saúde Materna e Infantil, Recife, v.9, n.3, p.331-8, 2009. CONTI, M.A.; FRUTUOSO, M.F.P.; GAMBARDELLA, A.M.D. Excesso de peso e insatisfação corporal em adolescentes. Revista de Nutriçáo, Campinas, v.18, n.4, p.491-7, 2005.

DE BRUIN, A.P.; OUDEJANS, R.R.D.; BAKKER, F.C. Dieting and body image in aesthetic sports: a comparison of dutch female gymnnasts and non-aesthetic sport partcipants. Psychology of Sport and Exercise, Amsterdam, v.8, n.4, p.507-20, 2007.

DENOMA, J.M.H.; SCARINGI, V.; GORDON, K.H.; VAN ORDEN, K.A.; JOINER, T.E. Eating disorder symptoms among undergraduate varsity athletes: club athletes, independent exercisers and nonoexercises. International Journal of Eating Disorders, Hoboken, v.12, n.1, p.47-53, 2009.

FILAIRE, E.; ROUVEIX, M.; PANNAFIEUX, C.; FERRAND, C. Eating atitudes, perfectionism and body-esteem of elite male judoists and cyclists. Journal of Sports Science and Medicine, Bursa, v.6, n.1, p.50-7, 2007.

FORTES, L.S.; AMARAL, A.C.S.; CONTI, M.A.; FERREIRA, M.E.C. Qualidades psicométricas do Eating Attitudes Test (EAT-26) para a população adolescente masculina brasileira. Psicologia: Reflexão e Crítica, Porto Alegre, (Submetido). GOMES, R.; SILVA, L. Desordens alimentares e perfeccionismo: um estudo com atletas portugueses. Psicologia em Revista, Belo Horizonte, v.15, n.3, p.469-89, 2010.

HAUSENBLAS, H.A.; DOWNS, D.S. Comparison of body image between athletes and nonathletes: a meta-analytic review. Journal Applied Sports Psychology, Rome, v.13, n.1, p.323-39, 2001.

INTERNACIONAL SOCIETY FOR ADVANCEMENT FOR KINEANTHROPOMETRY (ISAK). International standards for anthropometric assessment. Sidney: National Library of Australia, 2001.

JOHNSON, C.; CROSBY, R.; ENGEL, S.; MITCHELL, J.; POWERS, M.; WITTROCK, D.; WONDERLICH, S. Gender, ethnicity, self-esteem and disordered eating among college athletes. Eating Behaviors, v.5, n.1, p.147-56, 2004. KRENTZ, E.M.; WARSCHBURGER, P. Sports-related correlates of disordered eating in aesthetic sports. Psychology of Sport and Exercise, Amsterdam, v.44, n.3, 315-21, 2011.

LEPAGE, M.L.; CROWTHER, J.H. The effects of exercise on body satisfaction and affect. Body Image, Amsterdam, v.7, n.1, p.124-30, 2010.

MARTINS, C.R.; PELEGRINI, A.; MATHEUS, S.C.; PETROSKI, E.L. Insatisfação com a imagem corporal e a relação com estado nutricional, adiposidade corporal e sintomas de anorexia e bulimia em adolescentes. Revista de Psiquiatria, Rio de Janeiro, v.32, n.1, p.19-23, 2010.

PERINI, T.A.; OLIVEIRA, G.L.; ORNELLAS, J.S.; OLIVEIRA, F.P. Cálculo do erro técnico de medição em antropometria. Revista Brasileira de Medicina do Esporte, São Paulo, v.11, n.1, 2005. 
PERINI, T.A.; VIEIRA, R.S.; VIGÁRIO; P.S.; OLIVEIRA, G.L.; ORNELLAS, J.S.; OILVEIRA, F.P. Transtorno do comportamento alimentar em atletas de elite de nado sincronizado. Revista Brasileira de Medicina do Esporte, São Paulo, v.15, n.1, p.54-7, 2009.

REIKING, M.F.; ALEXANDER, L.E. Prevalence of disordered-eating behaviors in undergraduate female collegiate athletes and nonathletes. Journal of Athletic Training, Dallas, v.40, n.1, p.47-51, 2005.

SCHAAL, K.; TAFFLET, M.; NASSIF, H.; THIBAULT, V.; PICHARD, C.; ALCOTTE, M.; GUILLET, T.; BERTHELOT, G.; SIMON S.; TOUSSAINT, J. Psychological balance in high level athletes: gender-based differences and sport-specific patterns. PLoS one, San Francisco, v.6, n.5, p.1-9, 2011.

SCHERER, F.C.; MARTINS, C.R.; PELEGRINI, A.; MATHEUS, S.C.; PETROSKI, E.L. Imagem corporal em adolescentes: associação com a maturação sexual e sintomas de transtornos alimentares. Jornal Brasileiro de Psiquiatria, Rio de Janeiro, v.59, n.3, p.198-202, 2010.

SCOFIER, S.; MAIANO, C.; D`ARRIPE-LONGUEVILLE, F. The effects of social relationships and acceptance on disturbed eating attitudes in elite adolescent female athletes: the mediating role of physical self-perceptions. International Journal of Eating Disorders, Hoboken, v.43, n.1, p. 65-71, 2010.

SLADE, P.D. Body image in anorexia nervosa. British Journal Psychiatry Supplement, London, v.2, n.1, p.20-2, 1988. SLAUGHTER, M.H.; LOHMAN, T.G.; BOILEAU, R.; HOSWILL, C.A.; STILLMAN, R.J.; YANLOAN, M.D. Skinfold equations for estimation of body fatness in children and youth. Human Biology, Baltimore, v.60, n.3, p.709-23, 1988. SUNDGOT-BORGEN, J.; TORSTVEIT, M. K. Prevalence of disorders in elite athletes in higher than in the general population. Clinical Journal of Sport Medicine, New York, v.14, n.1, p.25-32, 2004.

TORSTVEIT, M.K.; SUNDGOT-BORGEN, J. The female athlete triad exists in both elite athletes and controls. Medicine and Science in Sports and Exercise, Madison, v.37, n.1, p.1449-59, 2005.

VIEIRA, L.F; OLIVEIRA, L.P.; VIEIRA, L.F.; VISSOCI, J.R.N.; HOSHINO, E.F.; FERNANDES, S.L. Distúrbios de atitudes alimentares e sua relação com a distorção da auto-imagem corporal em atletas de judô do estado do Paraná. Revista da Educação Física/UEM, Maringá, v.17, n.2, p.177-84, 2006.

VIEIRA, J.L.L.; AMORIM, H.Z.; VIEIRA, L.F.; AMORIM, A.C.; ROCHA, P.G.M. Distúrbios de atitudes alimentares e distorção da imagem Corporal no contexto competitivo da ginástica rítmica. Revista Brasileira de Medicina do Esporte, São Paulo, v.15, n.6, p.410-4, 2009.

WORLD HEALTH ORGANIZATION (WHO). Development of a WHO growth reference for school-aged children and adolescents. Bulletin of the World Health Organization, Geneve, v.85, n.9, p.660-7, 2007.

\begin{tabular}{r|l} 
ENDEREÇo & \\
Leonardo de Sousa Fortes & \\
R. Guaçui, 525/202 & Recebido para publicação: 10/08/2011 \\
uiz de Fora - MG - BRASIL & Revisado: 24/ 10/2011 \\
& Aceito: 26/10/2011 \\
&
\end{tabular}

716 • Rev. bras. Educ. Fís. Esporte, São Paulo, v.25, n.4, p.707-16, out./ dez. 2011 